\title{
A Critical Case Report-Cloacal Malformation: Prenatal Diagnosis and Autopsy Findings
}

\section{Janak Desai', Ami Mehta', Purva Patel', Vishal Chavda $^{2 *}$ \\ ${ }^{1}$ Department of Obstetrics and Gynaecology, Division of Fetal Medicine, Sheth VS General Hospital, India ${ }^{2}$ Department of Pharmacology, Nirma University, India *Corresponding author: Vishal Chavda, Research Scholar (Neuropharmacology/ Neurology), Department of Pharmacology, Nirma University, Gujarat, India}

Received: December 06, 2018; Accepted: J anuary 11, 2019; Published: January 18, 2019

\begin{abstract}
Objective: Cloacal anomalies comprising rare pathological conditions which are observed in female fetuses and must be diagnosed at an early stage of pregnancy.

Case Report: An antenatal patient, 23 years old, G3P2L2 was referred to our obstetric service with an ultrasound report of singleton pregnancy of 37 weeks with a septated cystic pelvic mass $(7.4 \mathrm{~cm} \times 7.3 \mathrm{~cm} \times 8.3 \mathrm{~cm})$ in the fetal abdomen at the region of the bladder, atrioventricular septal defect and bilateral renal agenesis with severe oligohydramnios. The patient gave a normal birth with $1.6 \mathrm{~kg}$ baby body weight. The newborn had abdominal distension, ambiguous genitalia, and rectal Artesia with a single opening at the perineum. The newborn was placed in the neonatal intensive care unit where further diagnostic investigations were conducted. The neonate died on the $1^{\text {st }}$ day after 5 hours of life due to respiratory distress. Autopsy and karyotyping were done with proper verbal and written consent of the guardian.
\end{abstract}

Conclusion: In our case, we reported a patient with a cloacal malformation with a cystic pelvic mass, fluid debris was seen on prenatal ultrasound which septate representing hydrocolpos of duplicating vagina. Thus, meconium and vaginal secretions accumulate in the compliant structure of vagina causing hydrocolpos which are rare condition rather than fetal ascites. Ultrasound with differential diagnosis at the early gestational stage can make a huge difference in decreasing such anomalies and early stage fetal mortalities.

\section{Introduction}

Cloacal anomalies comprise of rare pathological conditions which are observed in female fetuses [1,2]. They present in various forms depending on the kind of malformation and the gestational age, at the time of diagnosis. The observed female prevalence is 1 in 50,000 births for the most common forms and 1 in 2,50,000 births for the rarest forms, that is cloacal extrophy $[1,3]$. The cloacal malformation is a condition in which there is a single opening for the genital, urinary and gastrointestinal tracts. Embryologically, this confluence normally persists only up to the fifth week of pregnancy $[1,4]$. Persistence of a cloacal condition is caused by abnormal development of the urorectal septum. Most of the cases described till date, have been diagnosed in the $3^{\text {rd }}$ trimester or immediately after birth. The discovery of a mega cyst during the 1st trimester may indicate a diagnosis of cloacal malformation, but the ultrasound imaging is mostly inconclusive especially in the 3rd trimester. Magnetic Resonance Imaging (MRI) has the capability to provide a more accurate diagnosis. Here, we present a critical case of cloacal anomaly, in which diagnostic suspicion came from obstetric Ultrasound (USG). Ultrasound findings were a cystic septated pelvic mass, atrioventricular septal defect, bilateral renal agenesis, no visualization of a bladder and these findings were confirmed by autopsy. MRI was not done because when the patient came she was in labour pain.

\section{Case Presentation}

An antenatal patient, 23 years old, G3P2L2 was referred to our

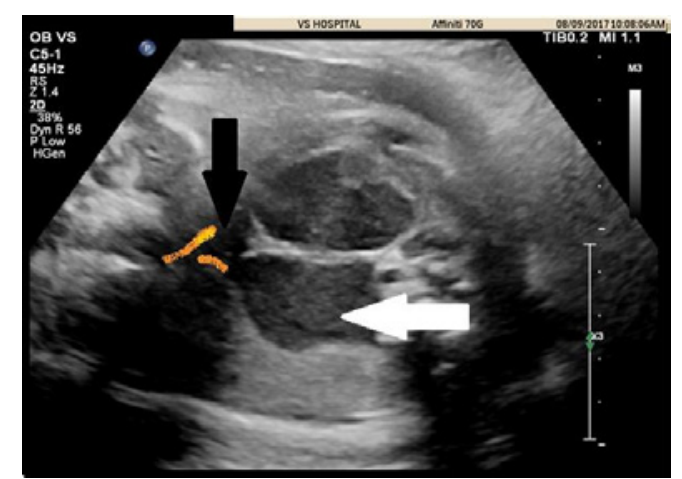

Figure 1: Prenatal sonogram shows a cystic pelvic mass with linear septation and fluid filled level representing duplicated obstructed vaginas consistent with progressive hydrocolpos(white arrow) and decompression of urinary bladder(black arrow).

obstetric service with an ultrasound report of singleton pregnancy of 37 weeks with a septated cystic pelvic mass. Our findings were a large septated cystic pelvic mass $(7.4 \mathrm{~cm} \times 7.3 \mathrm{~cm} \times 8.3 \mathrm{~cm})$ in the fetal abdomen in the region of the bladder, atrioventricular septal defect and bilateral renal agenesis. The bladder was not seen separately. There was severe oligohydramnios. Umbilical Doppler PI was- 1.2. Patient delivered vaginally, a baby of $1.6 \mathrm{~kg}$ weight. The newborn had abdominal distension, ambiguous genitalia (short penile like structure and fused labia majora) and rectal atresia. There was a single opening on the perineum between the labia majora like structure.
Austin J Surg - Volume 6 Issue 2 - 2019

ISSN : 2381-9030 | www.austinpublishing group.com

Chavda et al. ( ) All rights are reserved
Citation: Desai J, Mehta A, Patel P, Chavda V. A Critical Case Report-Cloacal Malformation: Prenatal Diagnosis and Autopsy Findings. Austin J Surg. 2019; 6(2): 1162 


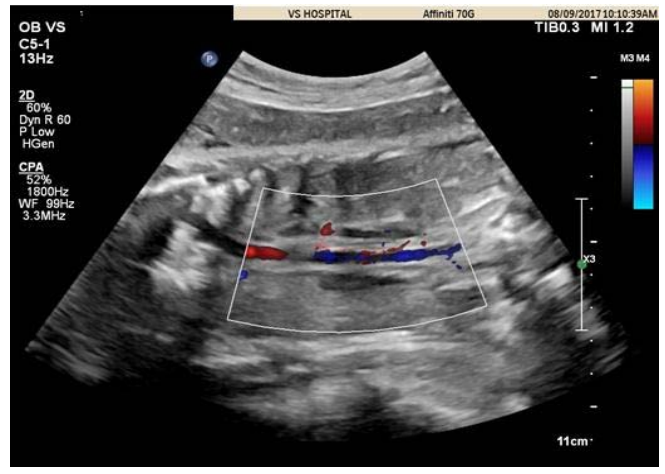

Figure 2: Bilateral renal agenesis with lying down adrenals.

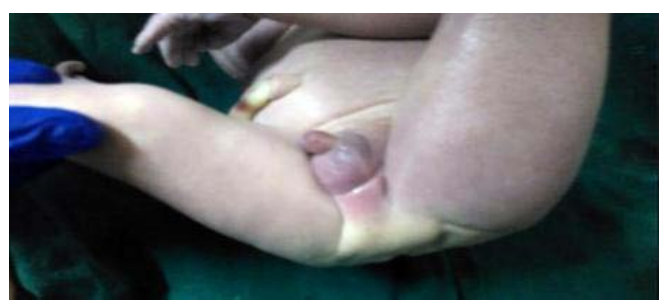

Figure 3: Ambiguous genitalia (fused labia majora, short penile like structures).

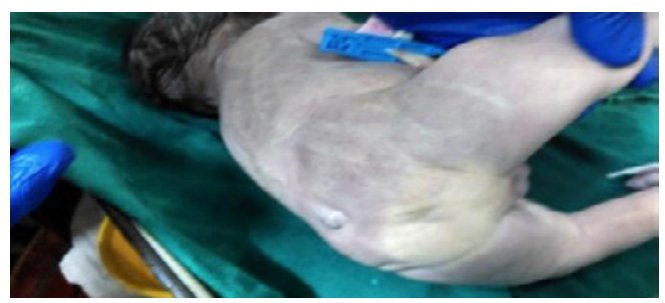

Figure 4: Anal aresia.

The newborn was placed in the neonatal intensive care unit where further diagnostic investigations were conducted. Pelvic ultrasound was done, the scan revealed a large retrovesical dense cystic mass with a median septum in the pelvic region and bilateral renal agenesis. The neonate died on the 1st day after 5 hours of life due to respiratory distress.

After obtaining informed consent from parents, an autopsy was done which revealed a single cystic pelvic mass in which all three urinary, anal and vaginal routes coalesced. Two separate uterine buds along with fallopian tube and ovary attached to it were seen on either side of the mass. Bilateral adrenals were seen lying vertically on either side of the vertebral column, both kidneys were absent. Small tissue of fetal tissue was sent for karyotyping which turned out to be normal.

\section{Discussion}

\section{Embryology}

In 4-5 weeks of an embryo, the cloaca is a common chamber of caudal intestinal, urinary and genital tracts. These structures give rise to urogenital sinus anteriorly and anorectum posteriorly. The urorectal membrane divides the urogenital sinus and anorectum at the cranial aspect of the cloaca. The separate anterior urogenital

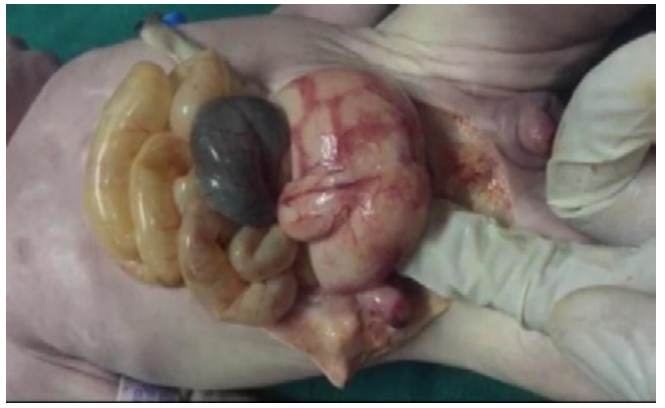

Figure 5: Cloacal cyst.

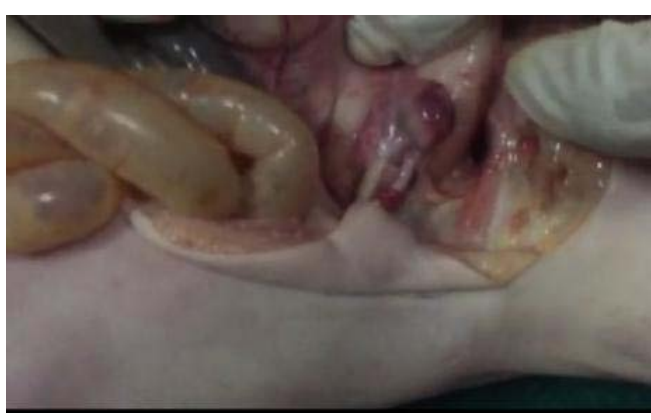

Figure 6: One sided uterine bud.

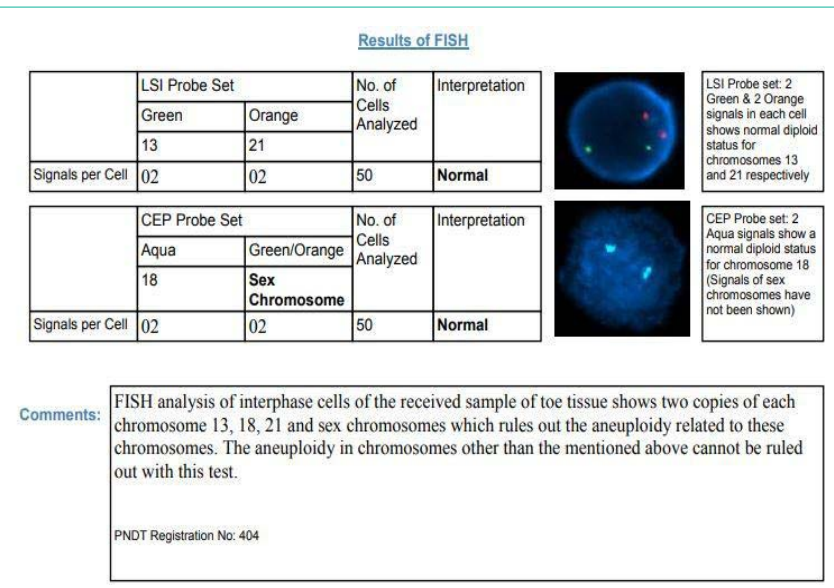

Figure 7: Fish results.

sinus and posterior anorectum then open through the perineum via dissolution of cloacal membrane once it comes into contact with the urorectal membrane. Urogenital sinus then becomes urinary bladder and urethra with a portion incorporate into the vagina and hymen [4].

\section{Etiology}

The spectrum of anomalies observed results from the lining of developmental arrest. The cause of arrest is not known however, recent evidence implicates homeobox and sonic hedgehog signaling rather than the teratogenic or hereditary cause. There are also strong hormonal influences proved by the association of urogenital malformation with virilization unrelated to congenital adrenal hyperplasia in females. The common observation of uterine and vaginal duplication has led to speculation that malformation of 
cloaca or urogenital sinus interferes with Mullerian duct fusion. Terminology in literature confusing, it reflects the range of anatomy observed and the proposed mechanisms of malformation. For example, the partial urorectal malformation sequence is synonyms with cloaca. The multitude of terms in literature makes it difficult to develop a useful classification system.

Prenatal diagnosis of female urogenital anomalies is usually difficult [5-7]. These anomalies are rare, presenting as varying defects, particularly in late stages of pregnancy lacking characteristics of any ultrasound signs. Cloacal anomalies result from failure of development of the urorectal fold, which separates the anorectal canal from the urogenital tract. This results in different degrees of anomalies ranging from female hypospadias, duplex uteri, bladder diverticulum, double vagina to more complex anomalies.

In this case, cystic pelvic mass with fluid debris was seen on prenatal ultrasound. The cystic mass was septate representing hydrocolpos of duplicating vagina. Thus, meconium and vaginal secretions accumulate in the compliant structure the vagina causing hydrocolpos. The common channel is virtually always present but there is a variable obstruction at possibly related to an angle at which it meets perineum.

In literature cases of cloacal anomaly have been reported to be associated with fetal ascites [8]. Fetal urine would drain through the fallopian tube to the peritoneal cavity; this process would develop a chemical reaction that would determine the tubal obstruction, hydrocolpos, and resolution of ascites but in our case no ascites was observed because of bilateral renal agenesis and it is very rare as till now only a few cases in number has been reported. Although ultrasonography is able to identify the large abdominal cystic mass, its origin cannot be determined in most cases. The differential diagnosis suggests-bowel atresia, fetal ovarian cyst, nonimmune hydrops, mega cystitis microcolon intestinal hypo-peristalsis syndrome and obstructive uropathy. In this case the presence of an abdominal septated cyst in the central region of the fetal pelvis confirmed the finding of vaginal duplication with hydrocolpos, thus increasing suspicion of a cloacal anomaly formed which was confirmed by autopsy. Thus differential diagnosis can make a huge difference if diagnosed at early stage of pregnancy with MRI, VCUG or genito/ sinogram, or cystoscopy and/or vaginoscopy [9-11]. It is important to diagnose with ultrasound such kind of malformation to prevent such rare anomalies to decrease such late stage morbidities.

\section{FISH study results}

The patient was an emergency case and we had to rule it out as per emergency diagnosis, in that particular we were suspecting trisomy 13, trisomy 18 so FISH had been sent with toe tissue from the foetus. Autopsy was performed after 20 hours so we could not get live tissue for karyotyping hence it wasn't performed. The results of FISH study indicated two copies of each chromosome 13, 18, 21 and sex chromosomes, which rules out the aneuploidy Figures 1-7.

\section{Ethical approval}

The patient was being brought to the gynaecology department in emergency. The patient was a minor as the procedure performed (Autopsy) was clearly explained in verbal, written and native understandable language for autopsy procedure and FISH study for future directions. As the fetous and mother were medical subjects, the proper consent was taken from both mother, his husband and related guardians to perform the procedure. They were also informed related to the publication of case report as a support for continuous medical education.

\section{Acknowledgment}

Fellow and faculties of Fetal Medicine Unit, Residents and faculties of Department of Obstetrics and Gynaecology, Sheth VS General Hospital.

\section{Clinical Significance}

Ultrasound with differential diagnosis at the early gestational stage can make a huge difference in decreasing such cloacal anomalies and early stage fetal mortalities.

\section{References}

1. Gupta P, Kumar S, Sharma R, Gadodia A. Case report: antenatal MRI diagnosis of cloacal dysgenesis syndrome. The Indian journal of radiology \& imaging. 2010; 20: 143.

2. Gupta R, Sharma P, Shukla AK, Goyal M, Gupta A. Cloacal malformation variant in a male neonate. Journal of Indian Association of Pediatric Surgeons. 2018; 23: 106.

3. Waters EG. Cloacal dysgenesis: related anomalies and pregnancies. Obstetrics and Gynecology. 1982; 59: 398-402.

4. Stephens FD. Embryology of the cloaca and embryogenesis of anorecta malformations. Birth Defects Orig Artic Ser. 1988; 24: 177-209.

5. Morikawa M, Yamada T, Cho K, Yamada H, Minakami H. Prenatal diagnosis and therapy of persistent cloaca: A case report. Fetal Diagn Ther. 2006; 21: 343-347.

6. Warne S, Chitty LS, Wilcox DT. Prenatal diagnosis of cloacal anomalies. BJU international. 2002; 89: 78-81.

7. Cilento BG, Benacerraf BR, Mandell J. Prenatal diagnosis of cloacal malformation. Urology. 1994; 43: 386-388.

8. Hamada T, Hirose R, Kosaka T, Taniguchi K, Noguchi M, Kihara T, et al. Giant cystic meconium peritonitis associated with a cloacal anomaly: case report. Journal of pediatric surgery. 2008; 43: e21-e23.

9. Ashour K, Shehata S, Osheba A. Cystourethroscopy versus contrast studies in urogenital sinus and cloacal anomalies in children. Journal of pediatric surgery. 2018; 53: 313-315.

10. Granberg CF, Gargollo PC. Neurogenic Bladder in a Girl after Surgery for Cloacal Malformation: The Importance of informing Patients and Parents of All Various Options. European urology focus. 2017; 3: 161-163.

11. Wagner G, Holschneider AM, Gharib M. A complex, high cloacal malformation: case report. European journal of pediatric surgery. 1998; 8: 182-185.
Austin J Surg - Volume 6 Issue 2 - 2019

ISSN : 2381-9030 | www.austinpublishing group.com

Chavda et al. (C) All rights are reserved
Citation: Desai J, Mehta A, Patel P, Chavda V. A Critical Case Report-Cloacal Malformation: Prenatal Diagnosis and Autopsy Findings. Austin J Surg. 2019; 6(2): 1162. 